\title{
Cloning of a caffeoyl-coenzyme A $O$-methyltransferase from Camellia sinensis and analysis of its catalytic activity"
}

\author{
Yue ZHANG, Hai-peng LV ${ }^{\dagger *}$, Cheng-ying MA, Li GUO, Jun-feng TAN, Qun-hua PENG, Zhi LIN ${ }^{\dagger *}$ \\ (Key Laboratory of Tea Biology and Resource Utilization of Ministry of Agriculture, \\ Tea Research Institute, Chinese Academy of Agricultural Sciences, Hangzhou 310008, China) \\ †E-mail: lvhaipeng@tricaas.com; linz@tricaas.com \\ Received July 8, 2014; Revision accepted Oct. 23, 2014; Crosschecked Jan. 8, 2015
}

\begin{abstract}
Epigallocatechin-3-O-(3-O-methyl) gallate (EGCG3"Me) present in leaves of Camellia sinensis has many beneficial biological activities for human health. However, EGCG3"Me occurs naturally in tea leaves in extremely limited quantities. Finding an enzyme from C. sinensis to catalyze the synthesis of EGCG3"Me is an alternative method to make up for the scarcity of EGCG3"Me in natural situations. In the present study, a complementary DNA (cDNA) encoding region and genomic DNA of the caffeoyl-coenzyme A O-methyltransferase (CCOAOMT) gene were isolated from C. sinensis (designated CsCCoAOMT). Nucleotide sequence analysis of CsCCOAOMT revealed an open reading frame of $738 \mathrm{bp}$ that encodes a polypeptide with a predicted molecular weight of $28 \mathrm{kDa}$, which correlated well with the results of sodium dodecyl sulfate-polyacrylamide gel electrophoresis (SDS-PAGE). The full-length DNA sequence (2678 bp) contained five exons and four introns. The deduced amino acid sequence of CsCCoAOMT shared $92 \%$ identity with CCOAOMTs from Codonopsis lanceolata and Betula luminifera. The catalytic activity of CsCCoAOMT was analyzed. Three monomethylated epigallocatechin-3-O-gallate (EGCG) compounds (EGCG4"Me, EGCG3"Me, and EGCG3'Me) were produced by CsCCoAOMT with $K_{\mathrm{m}}$ in the micromolar range. Real-time polymerase chain reaction (RT-PCR) experiments indicated that the CsCCOAOMT transcript was present at low levels during the early stages of leaf maturity (the first leaf and bud on a shoot) but the relative expression was augmented at advanced stages of leaf maturity (the third or fourth leaf on a shoot), which accorded well with changes in EGCG3"Me content in fresh leaves. Hence, we concluded that CsCCoAOMT catalyzes the syntheses of methylated EGCGs.
\end{abstract}

Key words: Tea (Camellia sinensis), O-methyltransferase, CsCCoAOMT, Prokaryotic expression, Catalytic activity, Methylated epigallocatechin-3-O-gallate (EGCG)

doi:10.1631/jzus.B1400193 Document code: A $\quad$ CLC number: S571.1

\section{Introduction}

Tea (Camellia sinensis) is consumed worldwide and its health-promoting effects have been widely

\footnotetext{
${ }^{\ddagger}$ Corresponding authors

* Project supported by the Zhejiang Provincial Natural Science Foundation of China (No. Y3080088), the National Natural Science Foundation of China (No. 30972404), the Earmarked Fund for China Agricultural Research System (No. CARS-23), and the Science and Technology Innovation Project of Chinese Academy of Agricultural Sciences (No. CAAS-ASTIP-2014-TRICAAS-OX)

(D) ORCID: Yue ZHANG, http://orcid.org/0000-0001-5793-6318; Hai-peng LV, http://orcid.org/0000-0002-4644-4323; Zhi LIN, http:// orcid.org/0000-0001-6738-6663

(C) Zhejiang University and Springer-Verlag Berlin Heidelberg 2015
}

explored (Lin and Lin-Shiau, 2006). Tea has many biological activities, such as anticancer (Jankun et al., 1997), anti-obesity (Kuo et al., 2005), and antibacterial properties (Bansal et al., 2013; Keller et al., 2013). Tea catechins are a group of polyphenolic compounds, which have been generally recognized as the major bioactive constituents responsible for the health properties of green tea. Epigallocatechin-3-O-gallate (EGCG), which accounts for almost $50 \%$ of the total catechins, shows the strongest biological activity in the vast majority of cases (Singh et al., 2011). Recently, however, it was reported that some $O$-methylated EGCGs occurring naturally in tea leaves, such as epigallocatechin-3-O-(3-O-methyl) 
gallate (EGCG3"Me) and epigallocatechin-3-O(4-O-methyl) gallate (EGCG4"Me), showed much stronger anti-allergic (Maeda-Yamamoto et al., 2004; 2007) and anti-hypertensive effects (Kurita et al., 2010) than EGCG. Moreover, $O$-methylated EGCG (for example, EGCG3"Me) was absorbed more easily than EGCG (Sano et al., 2000). Therefore, these $O$-methylated EGCGs have attracted much attention in recent years, especially EGCG3"Me. Unfortunately, EGCG3"Me occurs in extremely limited quantities, and very few tea genotypes contain $>1 \%$ EGCG3"Me. Consequently, increasing the supply of $O$-methylated EGCGs by extraction from tea tissues is difficult; therefore, it is necessary to find alternative methods for efficient synthesis of EGCG3"Me.

Methylation reactions occur ubiquitously in all organisms. It is well known that $O$-methyltransferases (OMTs) are extremely important enzymes in plants that have significant regulatory effects on growth and development. Plant OMTs are generally categorized into two families according to their distinct substrate preferences and amino acid sequence homology, namely PI-OMT I and PI-OMT II. The lengths of PI-OMT I proteins vary from 231 to 248 amino acids and their activities are dependent on the presence of divalent cations. The lengths of PI-OMT II enzymes are 344-383 amino acids and, unlike the PI-OMT I family, the activities of PI-OMT II enzymes do not require $\mathrm{Mg}^{2+}$ ions. Caffeoyl-coenzyme A $O$-methyltransferase (CCoAOMT) belongs to the PI-OMT I family (Joshi and Chiang, 1998). It is well known that CCoAOMT is a key enzyme in the biosynthesis of lignin and catalyzes the methylation of caffeoyl-CoA to feruloyl$\mathrm{CoA}$ in guaiacyl lignin biosynthesis. As an $S$-adenosylL-methionine (SAM)-dependent OMT, CCoAOMT transfers the methyl groups of SAM to the aromatic ring of monolignols (Zhao et al., 2004). Its amino acid sequence contains eight conserved motifs. Motifs A, $\mathrm{B}$, and $\mathrm{C}$ are common in OMTs, which are considered to be putative SAM-binding motifs, whereas motifs $\mathrm{D}, \mathrm{E}, \mathrm{F}, \mathrm{G}$, and $\mathrm{H}$ are the signature sequences of CCoAOMT (Hoffmann et al., 2001).

It has been reported that some of the CCoAOMT may utilize both flavonoids and caffeoyl-CoA (Kim et al., 2010a; 2010b). EGCG is a flavanol, which is a class of flavonoids. Consequently, CCoAOMT in plants probably shows catalytic activity in the methylation of EGCG. Kirita et al. (2010) cloned a novel
OMT from $C$. sinensis and several $O$-methylated EGCGs were synthesized by the recombinant enzyme. The enzyme was proposed to be a CCoAOMT. In the present study, a CCOAOMT gene in $C$. sinensis (CsCCoAOMT) was cloned and expressed in Escherichia coli. The gene structure, catalytic activity, and kinetic parameters of CsCCoAOMT were analyzed. Furthermore, we compared the relative expression level of CsCCoAOMT with EGCG3"Me content in fresh leaves of four tea cultivars.

\section{Materials and methods}

\subsection{Plant materials, plasmids, and standards}

Plant materials: four cultivars T1, T2, T3, and T4 grew in the tea plantation of the Tea Research Institute of the Guangdong Academy of Agricultural Sciences, China. Fresh leaf samples comprising four leaves and a bud were plucked from the four cultivars in July 2012. All leaf samples were immediately frozen in liquid nitrogen and stored at $-80^{\circ} \mathrm{C}$.

E. coli strains and plasmids: E. coli BL21 (DE3) and $E$. coli DH5 $\alpha$ (TransGen Biotech, China), pET-51b(+) vector (Novagen, USA), cloning vector pMD18-T (TaKaRa, China). All strains were grown in LuriaBertani (LB) medium.

Standards of EGCG3"Me, EGCG4"Me, and EGCG3'Me were kindly supplied by Taiyo Kagaku Co., Ltd. (Japan).

\subsection{Cloning of $\mathrm{CsCCoAOMT}$}

Total RNA was extracted from leaf samples using the TRIzol reagent in accordance with the manufacturer's instructions (Invitrogen, USA), and the integrity and quality of the RNA were checked according to Mamati et al. (2006). First-strand complementary DNA (cDNA) was synthesized using $1.0 \mu \mathrm{g}$ total RNA with SuperScript Reverse Transcriptase (Invitrogen, USA). Genomic DNA was extracted using a modified cetyltrimethylammonium bromide (CTAB) method (Khan et al., 2004). Two primers (CSF 5'-ATGGCAACAAACGGAGAAGG A-3' and CSR 5'-GCAGACACGCCGGCACAG-3'), used for polymerase chain reaction (PCR) amplification of the CsCCOAOMT cDNAs and genomic DNA, were designed and synthesized according to the published cDNA sequence (GenBank Accession No. 
DD361102). The PCR reactions were performed with Phusion DNA polymerase (Finnzymes, Finland) in three steps: (1) pre-denaturation at $95{ }^{\circ} \mathrm{C}$ for $5 \mathrm{~min}$; (2) 35 amplification cycles at $95{ }^{\circ} \mathrm{C}$ denaturation for $30 \mathrm{~s}$, annealing at $61{ }^{\circ} \mathrm{C}$ for $45 \mathrm{~s}$ and extension at $72{ }^{\circ} \mathrm{C}$ for $1 \mathrm{~min}$ (for cDNA amplification) or $3.5 \mathrm{~min}$ (for genomic DNA amplification); and, (3) postextension at $72{ }^{\circ} \mathrm{C}$ for $10 \mathrm{~min}$. The PCR products were analyzed in $1.5 \%(0.015 \mathrm{~g} / \mathrm{ml})$ agarose gel and the bands containing the target fragments were purified using the EasyPure Quick Gel Extraction Kit (TransGen Biotech, China). 'A' tails were added to the purified fragments.

The purified DNA fragments with ' $A$ ' tails were inserted into the cloning plasmid pMD18-T vector (TaKaRa, China) and mixed with T4 DNA ligase at $16{ }^{\circ} \mathrm{C}$ for $5 \mathrm{~h}$. All the connected products were inserted into the competence $E$. coli DH5 $\alpha$. The cultures were placed on ice for $30 \mathrm{~min}$, given a heat shock at $42{ }^{\circ} \mathrm{C}$ for $1 \mathrm{~min}$, and then transferred to an ice bath for 5 min, followed by culturing on solid LB medium with $100 \mu \mathrm{g} / \mathrm{ml}$ ampicillin at $37^{\circ} \mathrm{C}$ overnight.

After clones were detected with M13 primers, positive clones were selected and multiplied in liquid LB medium supplemented with ampicillin for $12 \mathrm{~h}$. Plasmid DNAs were extracted with the EasyPure Plasmid MiniPrep Kit (TransGen Biotech, China).

\subsection{Sequence analysis}

DNA sequencing was performed by Invitrogen, Shanghai, China. Sequence analyses were performed online (http://www.ncbi.nlm.nih.gov/). The BLAST and BLASTP programs were used for the sequence homology search. By comparison of the cDNA sequences with the genomic DNA sequences, the introns and exons of CsCCoAOMT were clear and definite. Amino acid sequences of CsCCoAOMT, CCoAOMT from 11 other plant species and CsOMT (GenBank Accession No. DD361102) were aligned using DNAMAN software.

\subsection{Prokaryotic expression and purification of CsCCoAOMT}

CsCCOAOMT was originally cloned in the pMD18-T plasmid. Based on the cDNA coding sequence of $C S C C O A O M T$, a pair of primers that contained two restriction sites, $K p n I$ and SalI, were designed. KpnI and SalI sites were introduced by PCR at the $5^{\prime}$ and $3^{\prime}$ ends of the $C S C C O A O M T$ sequence using the following primers: 5'-GGGGTACCCATGGCA ACAAACGGAGAAGGA-3' (CS51F, KpnI site underlined) and 5'-ACGCGTCGACGCAGACACGCC GGCACAG-3' (CS51R, SalI site underlined). The digested products were inserted into the expression plasmid pET-51b(+) mixed with T4 DNA ligase at $16{ }^{\circ} \mathrm{C}$ for $12 \mathrm{~h}$. The pET-51b(+) plasmid was also digested with KpnI and SalI. The connected products were transformed in E. coli BL21 (DE3) and cultured in LB medium at $37{ }^{\circ} \mathrm{C}$ overnight, then subjected to restriction enzyme digestion and sequencing analysis.

The cells containing pET-51b-CCoAOMT were selected and cultured in $\mathrm{LB}\left(\mathrm{Amp}^{+}\right)$medium for about $5 \mathrm{~h}$, then the culture liquid was added to $500 \mathrm{ml} \mathrm{LB}$ $\left(\mathrm{Amp}^{+}\right)$liquid medium in the proportion of 1:100 (v/v) for further culture. When the culture liquid reached an absorbance of 0.6 at $600 \mathrm{~nm}$, isopropyl $\beta$-D-1thiogalactopyranoside (IPTG) was added to a final concentration of $0.1 \mathrm{mmol} / \mathrm{L}$ to induce expression of the fusion protein. After $16 \mathrm{~h}$ of induction at $20^{\circ} \mathrm{C}$, the bacterial cells were collected, resuspended in histidine (His)-tag binding buffer $\left(20 \mathrm{mmol} / \mathrm{L} \mathrm{NaH}_{2} \mathrm{PO}_{4}\right.$, $500 \mathrm{mmol} / \mathrm{L} \mathrm{NaCl}, \mathrm{pH} \mathrm{7.4)}$, and lysed by sonication. Crude enzymes $(5 \mathrm{ml})$ were added into a His-tag affinity column (Amersham Biosciences, USA), which had been balanced with $20 \mathrm{mmol} / \mathrm{L}$ phosphate-buffered saline ( $\mathrm{pH}$ 7.4) for $10 \mathrm{~min}$. The His-tag affinity column was stored at $4{ }^{\circ} \mathrm{C}$ for $2 \mathrm{~h}$, then washed with an imidazole buffer concentration gradient $(0,10,20,30,40$, $50,60,70,80,90,100,200,300,400$, and $500 \mathrm{mmol} / \mathrm{L}$ ). The eluent was collected in different tubes and the absorbance rate determined at $280 \mathrm{~nm}$. Those tubes showing absorbance at $280 \mathrm{~nm}$ were selected, and the selected samples were analyzed by sodium dodecyl sulfate-polyacrylamide gel electrophoresis (SDS-PAGE) using $12 \%(0.12 \mathrm{~g} / \mathrm{ml})$ polyacrylamide gel. Thus, the optimal concentration for eluting the fusion protein was ascertained.

\subsection{Enzyme assays, product identification, and kinetic analysis}

Enzymatic reactions were performed using the purified recombinant protein. To evaluate the enzyme activity of CsCCoAOMT, $0.2 \mathrm{mg}$ purified protein was added to a $15-\mathrm{ml}$ reaction system including $0.05 \mathrm{mmol} / \mathrm{L}$ EGCG, $0.16 \mathrm{mmol} / \mathrm{L} \mathrm{SAM}, 0.2 \mathrm{mmol} / \mathrm{L}$ $\mathrm{MgCl}_{2}$, and $100 \mathrm{mmol} / \mathrm{L}$ Tris- $\mathrm{HCl}(\mathrm{pH}$ 6.8). The 
reaction mixture was incubated at $35^{\circ} \mathrm{C}$ for $30 \mathrm{~min}$, before the reaction was terminated by addition of $1 \mathrm{~mol} / \mathrm{L} \mathrm{HCl}$. After the mixture was extracted with $30 \mathrm{ml}$ ethyl acetate, the organic phase was evaporated to dryness, and the residue was resuspended in $1 \mathrm{ml}$ ultra-pure water. Kinetic analyses were performed within the linear range of reaction velocities by adjusting the concentrations of EGCG (50 to $500 \mu \mathrm{mol} / \mathrm{L}$ ) and SAM ( 80 to $400 \mu \mathrm{mol} / \mathrm{L}$ ). All the methylated EGCGs are used as products for determining the kinetic parameters.

The resuspended products were analyzed using an Agilent Infinity 1290 high-performance liquid chromatography (HPLC) equipped with an Agilent 6530 QTOF mass spectrometer with a dual electrospray ionization source in the positive ionization mode. A Waters SunFire C18 column $(150 \mathrm{~mm} \times 2.1 \mathrm{~mm}$ internal diameter, $5 \mu \mathrm{m}$ particle size) was selected as the stationary phase, and the column temperature was set at $40{ }^{\circ} \mathrm{C}$. The binary mobile phase was $0.5 \%$ formic acid solution including $2 \mathrm{mmol} / \mathrm{L}$ ammonium formate (A) and acetonitrile (B). The gradient elution began with $12 \%$ solvent $\mathrm{B}$, increased linearly to $25 \%$ solvent $\mathrm{B}$ over $5 \mathrm{~min}$, and continued to increase to $75 \%$ solvent $\mathrm{B}$ over 15 min before decreasing to $12 \%$ solvent B over $5 \mathrm{~min}$. The flow rate was $0.3 \mathrm{ml} / \mathrm{min}$ and the injection volume was $5 \mu$ l. The mass accuracy was controlled through online calibration using reference ions $(m / z 121.0509,922.0098)$. The capillary voltage, temperature, nebuliser pressure, and flow rate of the drying gas were set to $3.5 \mathrm{kV}, 300{ }^{\circ} \mathrm{C}, 35 \mathrm{psi}(1 \mathrm{psi}=$ $6.895 \mathrm{kPa}$ ), and $8 \mathrm{~L} / \mathrm{min}$, respectively. Mass spectra were recorded for the $m / z$ range of $100-1000$. For accurate identification, the methylated products were subjected to the accurate mass, mass patterns, and retention time comparison with authentic standards.

\subsection{Analysis of $\mathrm{CSCCoAOMT}$ expression and EGCG3"Me content}

The relative abundance of the CsCCOAOMT transcript was measured by real-time PCR (RT-PCR) at four stages of leaf maturity (the first leaf and bud, the second leaf, the third leaf, and the fourth leaf from the shoot apex) of four tea cultivars (T1, T2, T3, and T4) that differed greatly in EGCG3"Me content in fresh leaves. The primers P3-R (reverse, 5'-ACACT GGTTACTCTCTTCTCG-3') and P3-F (forward, 5'-GATGTAGTTGTCCTTGTCAGC-3') were de- signed on the basis of the cDNA sequences. In addition, the 18S rRNA gene, amplified with the primers 18S rRNA-R (reverse, 5'-GCTGGAATTACCGCGG CT-3') and 18S rRNA-F (forward, 5'-CGGCTACCA CATCCAAGGAA-3'), was used as an internal reference gene.

RT-PCR was performed with the 7500 Real-Time PCR System (Applied Biosystems, Inc., CA, USA) with the following settings: $95^{\circ} \mathrm{C}$ for $60 \mathrm{~s} ; 40$ cycles of $95^{\circ} \mathrm{C}$ for $30 \mathrm{~s}, 60^{\circ} \mathrm{C}$ for $34 \mathrm{~s}$, and $72{ }^{\circ} \mathrm{C}$ for $30 \mathrm{~s}$. PCR reactions were carried out in triplicate on a 96-well plate with $50 \mu$ l per well using SYBR Premix Ex Taq (TaKaRa, China). The relative quantification values for each gene were calculated with the $\Delta \Delta C_{\mathrm{T}}$ method (Livak and Schmittgen, 2001; Ma et al., 2012). Data represent the average of three experiments.

The EGCG3"Me contents in leaves at four stages of maturity for each of the four tea cultivars were detected by HPLC according to the methods of Lv et al. (2008). A Waters 2489-2690 HPLC was used and samples were separated using a Waters SunFire C18 column $(150 \mathrm{~mm} \times 2.1 \mathrm{~mm}, 5 \mu \mathrm{m})$.

\section{Results}

\subsection{Isolation of CsCCoAOMT gene}

The cDNA (GenBank Accession No. JQ790527) and genomic DNA (GenBank Accession No. JQ790528) of CsCCOAOMT gene were cloned. Sequence analysis of CSCCOAOMT showed that the open reading frame (ORF) contained 738 bp (Fig. 1a), which encoded a polypeptide of 245 amino acid residues (Fig. 2). The genomic DNA of CsCCOAOMT was 2678 bp in length and comprised five exons and four introns (Figs. 1b and 1c), which was in concordance with the maize and Eragrostis curvula CCOAOMT1 gene intron-exon arrangement. In contrast, three introns were identified in rice CCOAOMT and maize CCOAOMT2 (Guillet-Claude et al., 2004).

\subsection{Sequence analysis of CsCCoAOMT}

Amino acid sequences of CsCCoAOMT, CCoAOMT proteins from 11 plant species, and CsOMT from $C$. sinensis were aligned using DNAMAN software. High homology between CsCCoAOMT and the other CCoAOMT proteins was apparent; for example, the predicted CsCCoAOMT 
protein sequence showed $92 \%$ identity with CCOAOMT proteins from Codonopsis lanceolata and Betula luminifera, and $87 \%$ similarity with CCoAOMT proteins from Gossypium hirsutum and Pinus pinaster. Homology between CsCCoAOMT and CsOMT from $C$. sinensis was $99 \%$. There were two amino acid differences between the CsCCoAOMT and CsOMT reported by Kirita et al. (2010). They were cloned from different tea genotypes. All regions were highly conserved except the $5^{\prime}$ end (Fig. 2). CsCCoAOMT contained all conserved motifs, i.e., the motifs A, B, C, D, E, F, G, and H. Motifs A, B, and $\mathrm{C}$ are common in plant OMTs and are thought to be specific SAM-binding sites. The specific spatial arrangement might be essential for the formation of the SAM-binding pocket (Joshi and Chiang, 1998; Zhao et al., 2004). Additional CCOAOMT signature motifs were indicated by D, E, F, G, and $\mathrm{H}$.

\subsection{Expression and purification of $\mathrm{CsCCoAOMT}$}

CsCCoAOMT was expressed successfully in E. coli strain BL21 (DE3) after induction of expression by IPTG for $16 \mathrm{~h}$. SDS-PAGE analysis showed that the relative molecular mass of the CsCCoAOMT fusion protein obtained was approximately $28 \mathrm{kDa}$, as expected, and was expressed both in the supernatant and sediment (Fig. 3a). After purification with a Histag affinity column and washing with an imidazole buffer concentration gradient, $200 \mathrm{mmol} / \mathrm{L}$ imidazole buffer was the optimal concentration for eluting the fusion protein. Hence, the target protein was collected

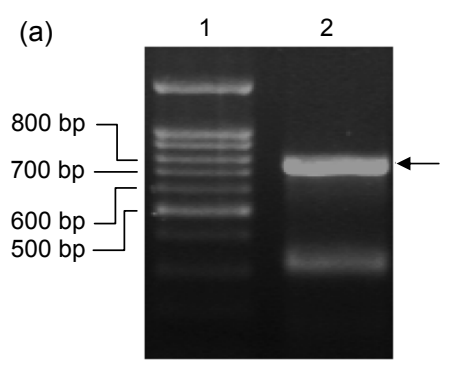

(c)

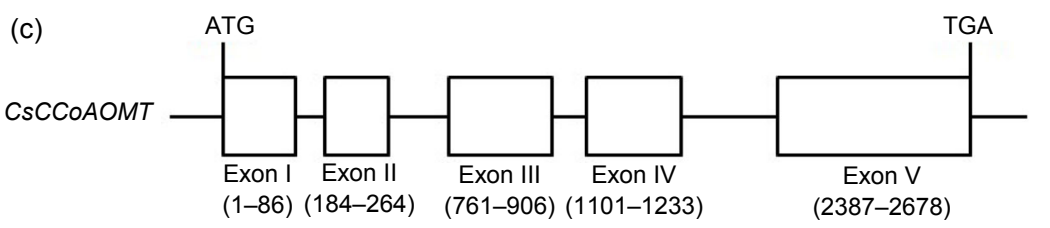

Fig. 1 Analysis of $C S C C O A O M T$ gene

The target products are indicated with black arrows. (a) Amplification of a cDNA ORF of CSCCoAOMT. 1, marker; 2, PCR product. (b) Amplification of genomic DNA of CsCCOAOMT. 1, marker; 2, PCR product. (c) Schematic representation of the structure of $C S C C O A O M T$. Five exons with their initial and final nucleotides are shown

in $200 \mathrm{mmol} / \mathrm{L}$ imidazole buffer, and then analyzed by SDS-PAGE (Fig. 3b). The purified enzyme was used to verify its catalytic activity.

\subsection{Catalytic activity and kinetic parameters of CsCCoAOMT}

The products of the enzymatic reaction (see Section 2.5) were analyzed by HPLC coupled to quadrupole time-of-flight mass spectrometry (HPLCQTOF-MS). No methylated products were detected in the control reactions (heated CsCCoAOMT, Fig. 4b), whereas with the addition of CsCCoAOMT, three monomethylated EGCG products were detected (Fig. 4a). The retention times $\left(t_{\mathrm{R}}\right)$ of these products (A, $\mathrm{B}, \mathrm{C}$ ) were $8.40,8.61$, and $9.10 \mathrm{~min}$ (Fig. 4a). Identical to the monomethylated EGCG standards, the $\mathrm{m} / \mathrm{z}$ of all three products was 473.1078, which was 14.1058 higher than the $m / z$ of EGCG (459.0020). From comparison of the ion chromatograms, it was clear that each $t_{\mathrm{R}}$ of products $\mathrm{A}, \mathrm{B}$ and $\mathrm{C}$ was consistent with the $t_{\mathrm{R}}$ of EGCG4"Me, EGCG3"Me, and EGCG3'Me, respectively (Fig. 4c). The MS patterns of the three products were also similar to those of the standards (Figs. 4d and 4e). The deviation between the actual and theoretical $\mathrm{m} / \mathrm{z}$ values of all detected compounds was less than $5 \times 10^{-6}$. These results indicated that the three products were identifiable as EGCG4"Me (compound A), EGCG3"Me (compound B), and EGCG3'Me (compound C). Therefore, it was concluded that CsCCoAOMT catalyzes the synthesis of methylated EGCG.
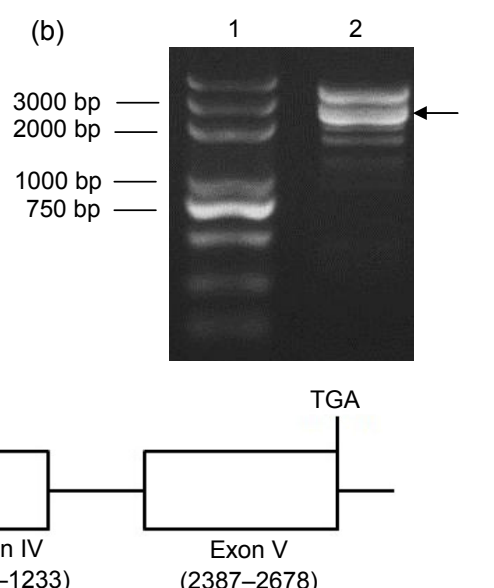


L. usitatissimum
N. tabacum
C. Ianceolata
P. pinaster
B. platyphylla
B. luminifera
P. bretschneideri
G. max
G. hirsutum
V. vinifera
P. lactiflora
C. sinensis
C. Sinensis (CSOMT)
Consensus

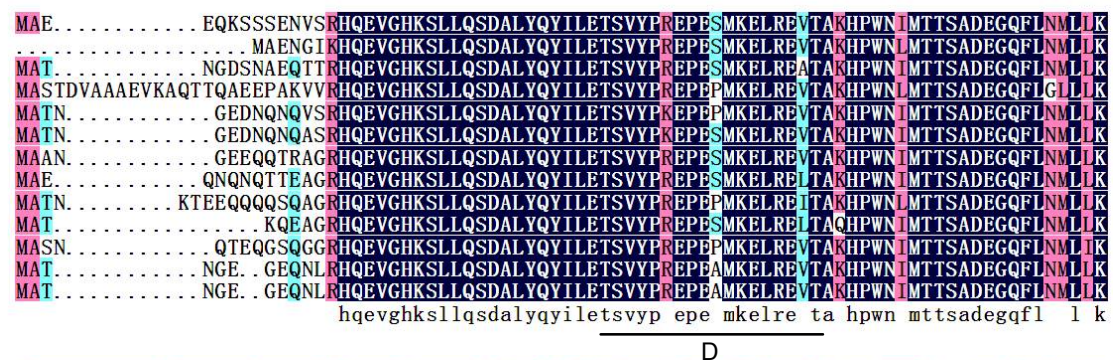

76
69
76
88
76
76
76
76
79
71
76
74
74

L. usitatissimum
N. tabacum
C. Ianceolata
P. pinaster
B. platyphylla
B. Iuminifera
P. bretschneideri
G. max
G. hirsutum
V. vinifera
P. lactiflora
C. sinensis
C. Sinensis (CsOMT)
Consensus

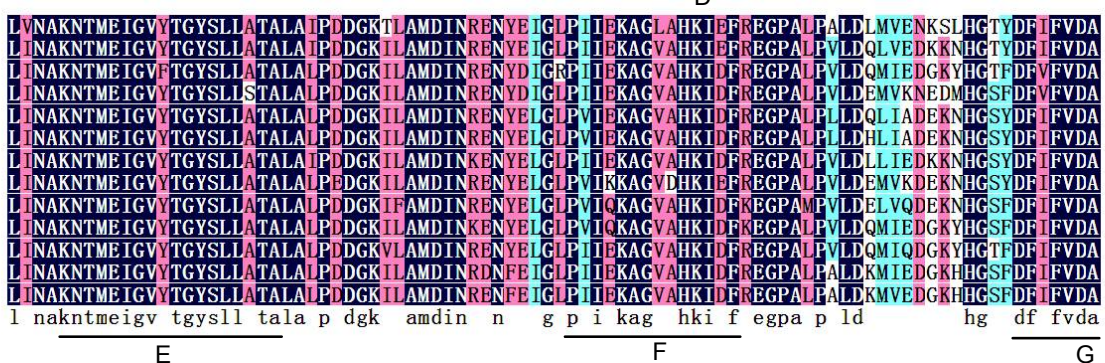

164

157

164
176

176
164

164
164

164
164

164
164
167

167
159

164
162

162

L. usitatissimum

N. tabacum

C. lanceolato

P. pinaster

B. platyphylla

B. luminifera
P. bretschneideri

P. bretsc
G. max

G. hirsutum

V. vinifera

P. lactiflora

C. sinensis

C. Sinensis
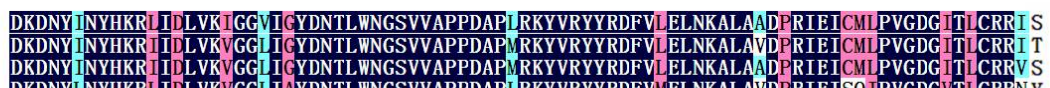

DNYLNYHKRLIDL

DKDNYINYHKRL IDL

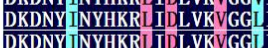

YDNTLWNGSVVAPPDAPMRKYVRYYRDFVLELNKALAADPRIEICMLPVGDG ITLCRRV S

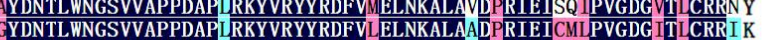

CDNTLWNGSVVAPPDAP LRKYVRYYRDFVLELNKALAADPRIEICMLPVGDG ITICRR I K

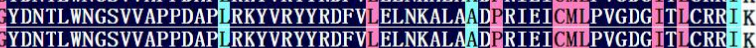

GDNTLWNGSVVAPPDAPLRKYVRYYRDFVLELNKALAADPRIEICMLPVGDG ITLCRR I K
YDNTLWNGSVVAPPDAPLRKYVRYYRDFVLELNKALAVDPRIEICMLPVGDG ITICRR I K

TDNYLNYHKRLIELVKVGGVI IGYDNTLWNGSVVAPPDAPLRKYVRYYRD

DKDNYLNYHKRIIDLVKVGG ITIGYDNTIM

DKDNYINYHKRLIDLVKV

YDNTLWNGSVVAPPDAPLRKYVRYY

.

dkdny nyhkr i lvk gg i ydntlwngsvaPPDAPMRYVRYYRDFVLELNKALAADPRIEICMLPVGGGGITLCRRV

A

B

$$
\begin{aligned}
& 247 \\
& 240 \\
& 247 \\
& 259 \\
& 247 \\
& 247 \\
& 247 \\
& 247 \\
& 250 \\
& 242 \\
& 247 \\
& 245 \\
& 245
\end{aligned}
$$

Fig. 2 Amino acid sequence alignment of CsCCoAOMT, CCoAOMT proteins from other plant species, and CsOMT from $C$. sinensis

Three putative SAM-binding motifs (A, B, and C) and additional CCoAOMT signature motifts (D, E, F, G, and H) are labeled. The sequences are from Codonopsis lanceolata (AB243012.1), Betula platyphylla (AY860952.1), Betula luminifera (FJ410449.1), Vitis vinifera (XM_002285070.1), Pyrus bretschneideri (JX457336.1), Linum usitatissimum (EU926495.1), Paeonia lactiflora (JQ684014.1), Glycine max (XM_003518654.1), Nicotiana tabacum (AF022775.1), Gossypium hirsutum (FJ848871.1), Pinus pinaster (FN824798.2), and Camellia sinensis (JQ790527). CsOMT is from Camellia sinensis (DD361102)
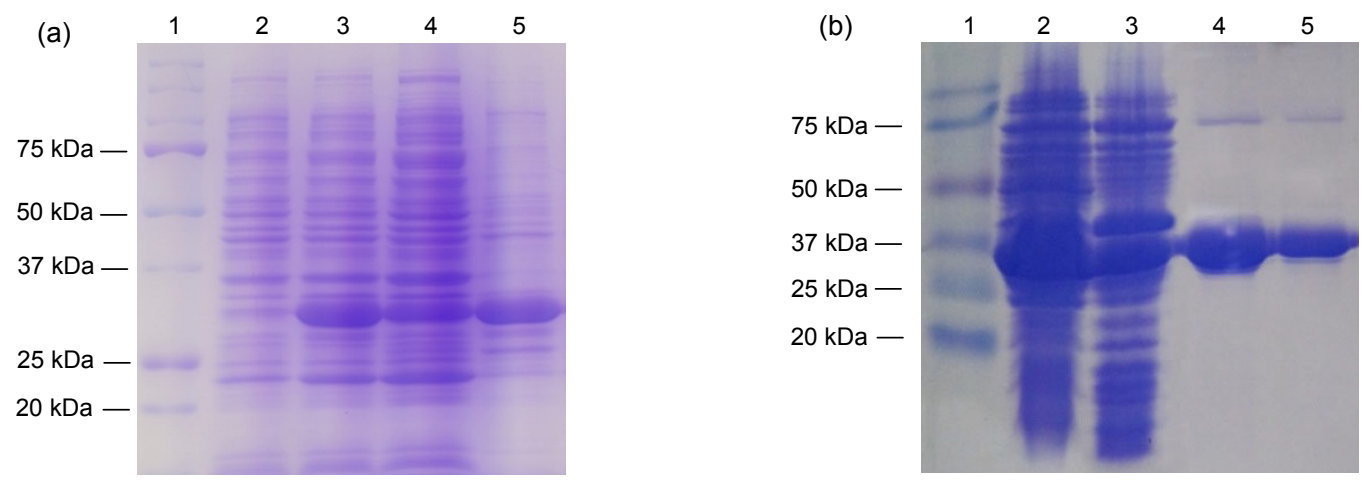

Fig. 3 Analysis of CsCCoAOMT expression and purification

(a) Analysis of prokaryotic expression of CsCCoAOMT by SDS-PAGE. 1, marker; 2, induction without IPTG; 3, total protein induced with IPTG for $16 \mathrm{~h}$; 4, soluble protein in the supernatant induced with IPTG for $16 \mathrm{~h}$; 5 , insoluble inclusion body in sediment induced with IPTG for $16 \mathrm{~h}$. (b) Analysis of purified CsCCoAOMT by SDS-PAGE. 1, marker; 2 and 3 , soluble protein of CsCCoAOMT without purification (as control); 4 and 5, soluble protein of CsCCoAOMT purified in $200 \mathrm{mmol} / \mathrm{L}$ imidazole eluant 


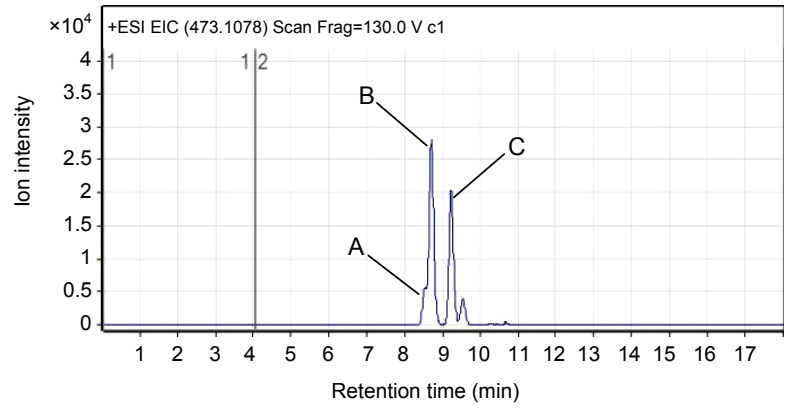

(a)

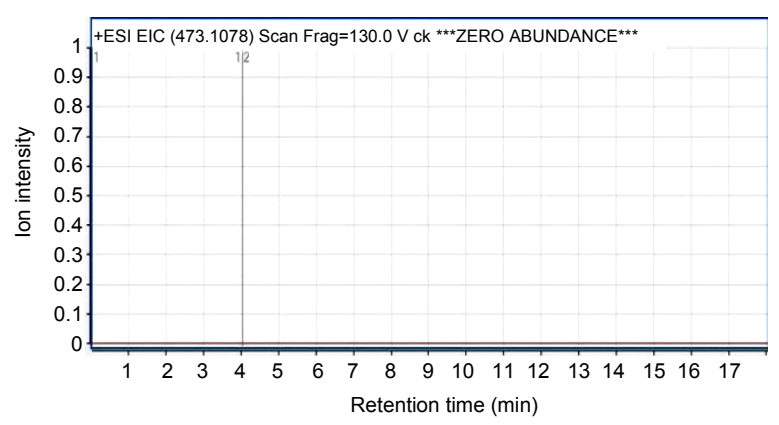

(b)

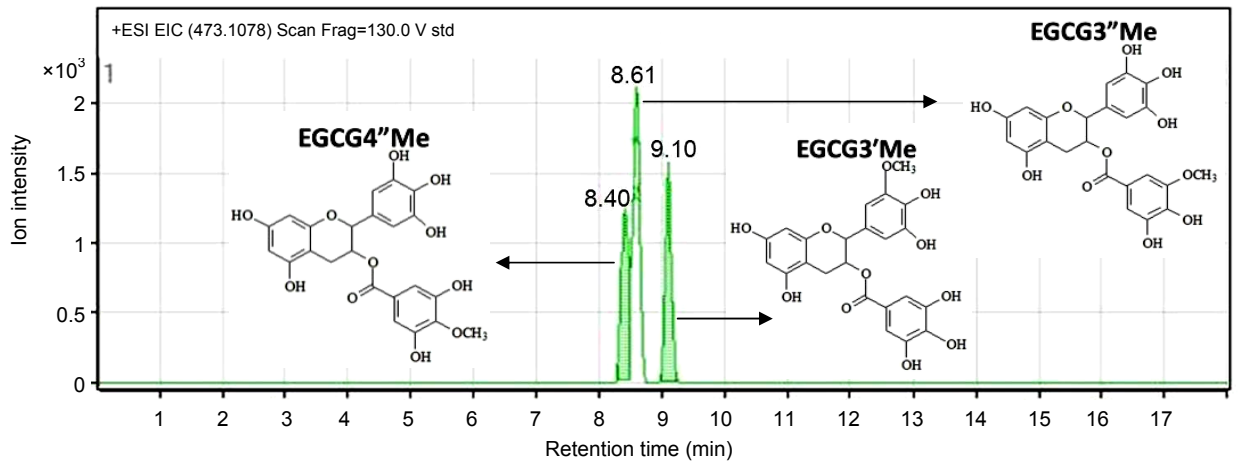

(c)
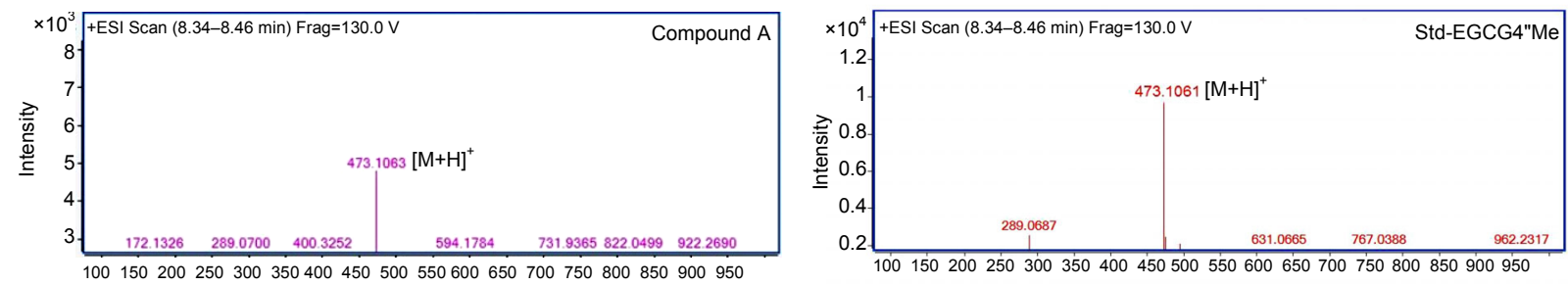

$\mathrm{m} / \mathrm{z}$
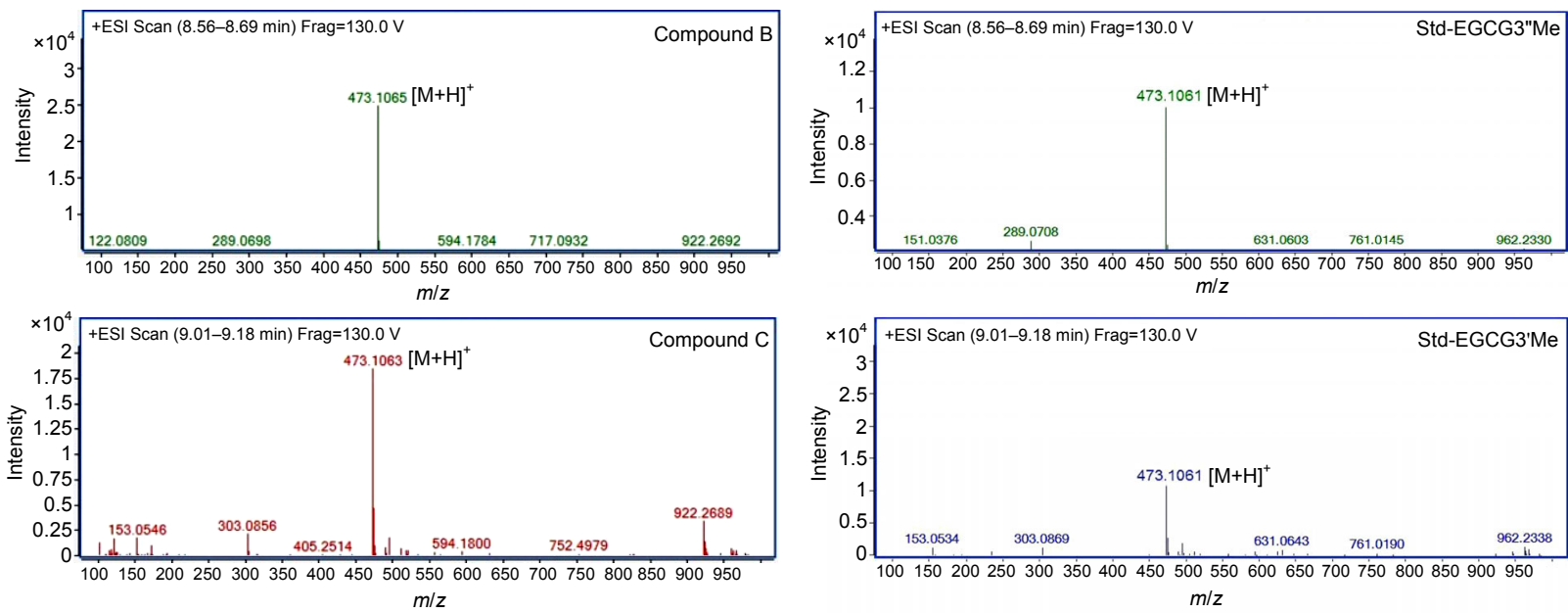

(d)

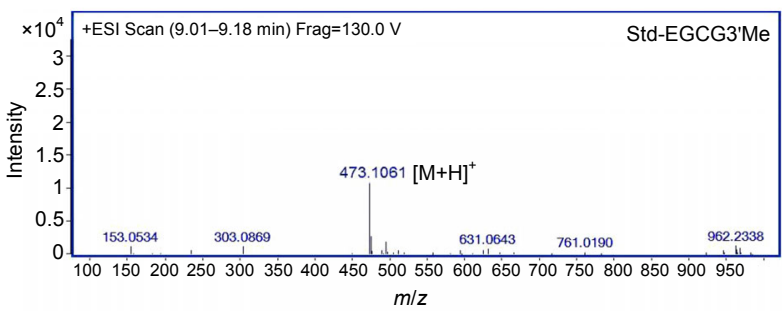

(e)

Fig. 4 Ion chromatogram and MS patterns for methylated products catalyzed by CsCCoAOMT and the monomethylated EGCG standards

(a) Ion chromatogram of monomethylated products catalyzed by CsCCoAOMT. (b) Ion chromatogram of control catalyzed by heated CsCCoAOMT. (c) Ion chromatogram for EGCG3"Me, EGCG4"Me, and EGCG3'Me standards. (d) MS patterns for compounds A, B, and C in (a). (e) MS patterns for the standards EGCG4"Me, EGCG3"Me, and EGCG3'Me. The LC-MS conditions are described in Section 2.5. The deviation of $\mathrm{m} / \mathrm{z}$ was less than $5 \times 10^{-6}$ 
The kinetic parameters of CsCCoAOMT were determined by reaction with EGCG as substrate, with SAM as the methyl donor (Table 1). CsCCoAOMT catalyzed the formations of EGCG3"Me, EGCG4"Me, and EGCG3'Me. All the three monomethylated EGCGs are used as products for determining the kinetic parameters. An apparent $K_{\mathrm{m}}$ value of $101 \mu \mathrm{mol} / \mathrm{L}$ and an apparent $K_{\text {cat }}$ value of $0.184 \mathrm{~s}^{-1}$ were measured for CsCCoAOMT with EGCG concentrations ranging from 50 to $500 \mu \mathrm{mol} / \mathrm{L}$. In reactions of CsCCoAOMT with the methyl donor SAM at concentrations ranging from 80 to $400 \mu \mathrm{mol} / \mathrm{L}$, CsCCoAOMT catalyzed the formation of methylated EGCG with an apparent $K_{\mathrm{m}}$ value of $160 \mu \mathrm{mol} / \mathrm{L}$ and an apparent $K_{\text {cat }}$ value of $0.049 \mathrm{~s}^{-1}$. The presented values are the averages of duplicate experiments.

Table 1 Kinetic parameters of CsCCoAOMT with EGCG as substrate

\begin{tabular}{ccccc}
\hline $\begin{array}{c}\text { Substrate/ } \\
\text { methyl } \\
\text { donor }\end{array}$ & $\begin{array}{c}V_{\max } \\
(\mu \mathrm{mol} /(\mathrm{mg} \cdot \mathrm{s}))\end{array}$ & $\begin{array}{c}K_{\mathrm{m}} \\
(\mu \mathrm{mol} / \mathrm{L})\end{array}$ & $\begin{array}{c}K_{\text {cat }} \\
\left(\mathrm{s}^{-1}\right)\end{array}$ & $\begin{array}{c}K_{\text {cat }} / K_{\mathrm{m}} \\
(\mathrm{L} /(\mathrm{mmol} \cdot \mathrm{s}))\end{array}$ \\
\hline EGCG & $0.29 \pm 0.02$ & $101 \pm 7.9$ & $0.184 \pm 0.02$ & 1.84 \\
SAM & $0.24 \pm 0.05$ & $160 \pm 18$ & $0.049 \pm 0.007$ & 0.31 \\
\hline
\end{tabular}

Data are expressed as mean \pm standard deviation (SD). $K_{\mathrm{m}}$, which is commonly known as Michaelis constant, is defined as the substrate concentration when the reaction velocity reaches half of the maximum $\left(V_{\max }\right) . K_{\text {cat }}$ expresses the number of substrate molecules turned over per enzyme molecule (or per activity center) per second; thus, $K_{\text {cat }}$ is sometimes called the turnover number

\subsection{Analysis of gene expression by RT-PCR}

Four tea cultivars (T1, T2, T3, and T4) that differing greatly in EGCG3"Me contents were selected to analyze the relative gene expression by RT-PCR. The mean contents of EGCG3"Me detected by HPLC in the four cultivars were $1.23 \%$ (T1), $0.82 \%$ (T2), $0.63 \%$ (T3), and $0.12 \%$ (T4), respectively. Semiquantitative expression analysis of CsCCoAOMT indicated that the gene was expressed in all of the cultivars. The relative expression level was high in the cultivar with the highest content of EGCG3"Me (T1), and intermediate in cultivars that accumulate moderate amounts of EGCG3"Me (T2 and T3), whereas in T4 EGCG3"Me was almost undetectable by HPLC and the relative expression level of $C s C C O A O M T$ was the lowest of the four cultivars (Fig. 5). Therefore, the relative gene expression levels in the four tea cultivars showed a tendency to increase consistent with increasing EGCG3"Me content in fresh leaves.
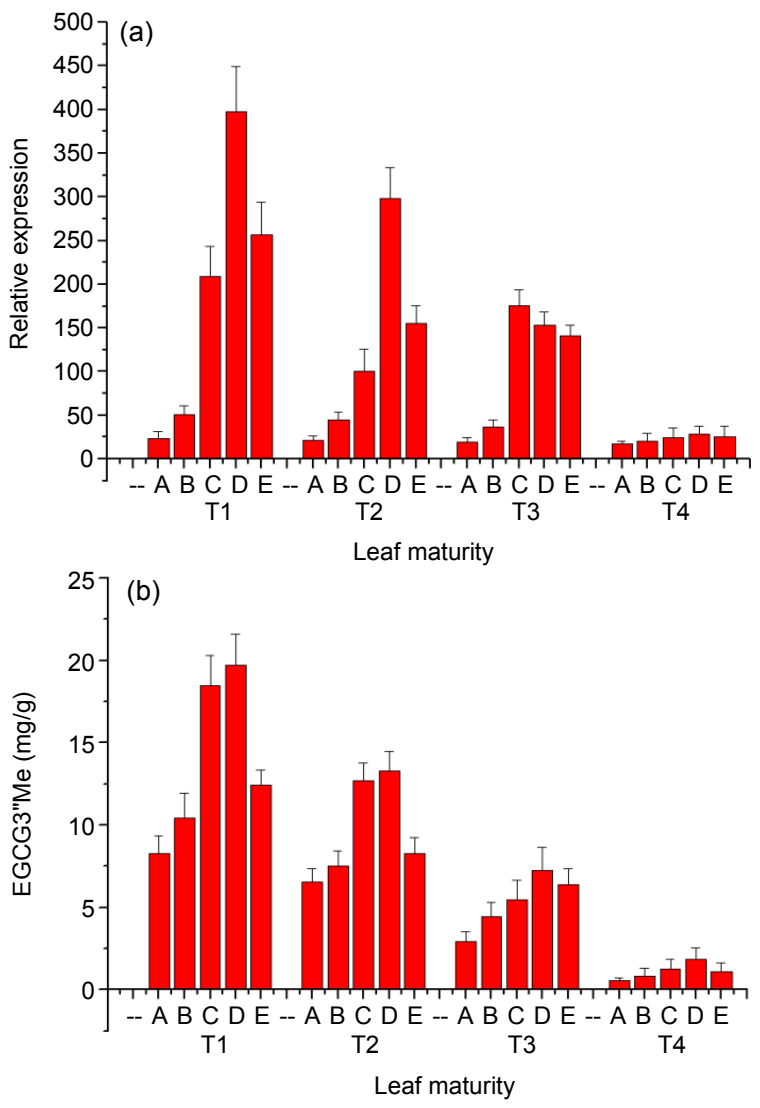

Fig. 5 Analysis of gene relative expression and EGCG3"Me content in leaves of four tea cultivars (T1, T2, T3, and T4)

A-D are different stages of leaf maturity: A, the first leaf and a bud; B, the second leaf; C, the third leaf; D, the fourth leaf; E: the mixed leaves above. (a) Relative expression of CsCCoAOMT quantified by RT-PCR; (b) EGCG3"Me content in leaves detected by HPLC

The relative expression level of CsCCoAOMT was further evaluated at different stages of leaf maturity (the first leaf and bud, the second leaf, the third leaf, and the fourth leaf from the shoot apex) in the four cultivars. The RT-PCR analysis demonstrated that rapid increase in transcript accumulation at the most advanced stages of maturity (the third leaf or fourth leaf), compared with the earliest stage (the first leaf and bud), indicating that the expression level of CsCCoAOMT was enhanced with increasing leaf maturity (Fig. 5a). There was a positive correlation between leaf maturity and EGCG3"Me content detected by HPLC (Fig. 5b), and with expression of CsCCOAOMT. These results indicated that CsCCOAOMT may be associated with biosynthesis of methylated catechin in $C$. sinensis. 


\section{Discussion}

In this study, genomic and cDNA clones for the CsCCOAOMT gene were isolated from $C$. sinensis. This is the first report of isolation of a genomic DNA clone for $C$. sinensis CCOAOMT. In addition, we obtained multiple CsCCOAOMT cDNA sequences (data not shown) that encoded four deduced amino acid sequences, in which a few differences were observed at several regular sites, but the homogeneity of the sequences was more than $99 \%$. The genetic background of $C$. sinensis is highly complex, so the reasons why there are sequence differences need further study. Moreover, the nucleotide and deduced amino acid sequences of CsCCoAOMT showed high homology with CCoAOMTs from 11 other plant species, which demonstrated that the gene is highly conserved among species and that the clone effectively codes for CCoAOMT. The alignment of the corresponding protein sequences from 11 other plant species (Fig. 2) showed $87 \%-92 \%$ homology with the C. sinensis-predicted protein sequence. The proposed structure for CsCCoAOMT consists of three putative SAM-binding motifs (A, B, and C) and five other regions $(\mathrm{D}, \mathrm{E}, \mathrm{F}, \mathrm{G}$, and $\mathrm{H})$ that may serve as CCoAOMT signatures in plants. Accordingly, the CsCCoAOMT amino acid sequence predicted here revealed the presence of the eight mentioned motifs (A-H) with conserved spatial relationships (Fig. 2).

As a methyl donor, SAM plays an important role in the formation of methylated EGCGs. We presumed that the number and type of methylated EGCG derivatives in the reaction mixture were related to the concentration of SAM. With increasing concentration of SAM, the catalysate became more complex and EGCG derivatives with multiple methyl groups were detected. It is reported that increasing the number of methyl groups of EGCG, especially in the galloyl moiety, helps it to acquire lipophilicity and show improved absorption and stability in animal and human plasma (Kirita et al., 2010). Therefore, we believe that the present study contributes effectively to the preparation of methylated EGCG. However, as is well known, CCoAOMT is a key enzyme in lignin biosynthesis and particularly in guaiacyl unit production. In the present experiments, CsCCoAOMT activity was not as high as we expected for catalyzing the synthesis of methylated EGCG. Therefore, a search for an EGCG-OMT that exhibits higher efficiency is required for efficient synthesis of EGCG3"Me.

\section{Compliance with ethics guidelines}

Yue ZHANG, Hai-peng LV, Cheng-ying MA, Li GUO, Jun-feng TAN, Qun-hua PENG, and Zhi LIN declare that they have no conflict of interest.

This article does not contain any studies with human or animal subjects performed by any of the authors.

\section{References}

Bansal, S., Choudhary, S., Sharma, M., et al., 2013. Tea: a native source of antimicrobial agents. Food Res. Int., 53(2):568-584. [doi:10.1016/j.foodres.2013.01.032]

Guillet-Claude, C., Birolleau-Touchard, C., Manicacci, D., et al., 2004. Genetic diversity associated with variation in silage corn digestibility for three $O$-methyltransferase genes involved in lignin biosynthesis. Theor. Appl. Genet., 110(1):126-135. [doi:10.1007/s00122-004-1808-4]

Hoffmann, L., Maury, S., Bergdoll, M., et al., 2001. Identification of the enzymatic active site of tobacco caffeoylcoenzyme A $O$-methyltransferase by site-directed mutagenesis. J. Biol. Chem., 276(39):36831-36838. [doi:10. 1074/jbc.M104977200]

Jankun, J., Selman, S.H., Swiercz, R., et al., 1997. Why drinking green tea could prevent cancer? Nature, 387(6633):561. [doi:10.1038/42381]

Joshi, C., Chiang, V.L., 1998. Conserved sequence motifs in plant $S$-adenosyl-L-methionine-dependent methyltransferases. Plant Mol. Biol., 37(4):663-674. [doi:10.1023/A: 1006035210889]

Keller, A.C., Weir, T.L., Broeckling, C.D., et al., 2013. Antibacterial activity and phytochemical profile of fermented Camellia sinensis (fuzhuan tea). Food Res. Int., 53(2): 945-949. [doi:10.1016/j.foodres.2013.04.023]

Khan, I.A., Awan, F.S., Ahmad, A., et al., 2004. A modified mini-prep method for economical and rapid extraction of genomic DNA in plants. Plant Mol. Biol. Rep., 22(1): 89a-89e. [doi:10.1007/BF02773355]

Kim, B.G., Sung, S.H., Chong, Y., et al., 2010a. Plant flavonoid $O$-methyltransferases: substrate specificity and application. J. Plant Biol., 53(5):321-329. [doi:10.1007/ s12374-010-9126-7]

Kim, B.G., Kim, D.H., Sung, S.H., et al., 2010b. Two $O$-methyltransferases from Picea abies: characterization and molecular basis of different reactivity. Planta, 232(4): 837-844. [doi:10.1007/s00425-010-1223-9]

Kirita, M., Honma, D., Tanaka, Y., et al., 2010. Cloning of a novel $O$-methyltransferase from Camellia sinensis and synthesis of $O$-methylated EGCG and evaluation of their bioactivity. J. Agric. Food Chem., 58(12):7196-7201. [doi:10.1021/jf100493s]

Kuo, K.L., Weng, M.S., Chiang, C.T., et al., 2005. Comparative studies on the hypolipidemic and growth suppressive 
effects of oolong, black, pu-erh, and green tea leaves in rats. J. Agric. Food Chem., 53(2):480-489. [doi:10.1021/ jf049375k]

Kurita, I., Maeda-Yamamoto, M., Tachibana, H., et al., 2010. Antihypertensive effect of Benifuuki tea containing $O$-methylated EGCG. J. Agric. Food Chem., 58(3):19031908. [doi:10.1021/jf904335g]

Lin, J.K., Lin-Shiau, S.Y., 2006. Mechanisms of hypolipidemic and anti-obesity effects of tea and tea polyphenols. Mol. Nutr. Food Res., 50(2):211-217. [doi:10.1002/ mnfr.200500138]

Livak, K.J., Schmittgen, T.D., 2001. Analysis of relative gene expression data using real-time quantitative PCR and the $2^{-\Delta \Delta C_{\mathrm{T}}}$ method. Methods, 25(4):402-408. [doi:10.1006/ meth.2001.1262]

Lv, H.P., Lin, Z., Tan, J.F., et al., 2008. Study on EGCG3"Me in tea. Food Ferment. Ind., 34(10):22-25 (in Chinese).

Ma, C.L., Chen, L., Wang, X.C., et al., 2012. Differential expression analysis of different albescent stages of 'Anji Baicha' (Camellia sinensis (L.) O. Kuntze) using cDNA microarray. Sci. Hort., 148(4):246-254. [doi:10.1016/j. scienta.2012.09.033]

Maeda-Yamamoto, M., Inagaki, N., Kitaura, J., et al., 2004. $O$-methylated catechins from tea leaves inhibit multiple protein kinases in mast cells. J. Immunol., 172(7):44864492. [doi:10.4049/jimmunol.172.7.4486]

Maeda-Yamamoto, M., Ema, K., Shibuichi, I., 2007. In vitro and in vivo anti-allergic effects of 'benifuuki' green tea containing $O$-methylated catechin and ginger extract enhancement. Cytotechnology, 55(2-3):135-142. [doi:10. 1007/s10616-007-9112-1]

Mamati, G.E., Liang, Y.R., Lu, J.L., 2006. Expression of basic genes involved in tea polyphenol synthesis in relation to accumulation of catechins and total tea polyphenols. J. Sci. Food Agric., 86(3):459-464. [doi:10.1002/jsfa.2368]

Sano, M., Miyase, T., Tachibana, H., et al., 2000. Antiallergic properties of tea components. Frag. J., 4:46-52 (in Japanese).

Singh, B.N., Shankar, S., Srivastava, R.K., 2011. Green tea catechin, epigallocatechin-3-gallate (EGCG): mechanisms, perspectives and clinical applications. Biochem. Pharmacol., 82(12):1807-1821. [doi:10.1016/j.bcp.2011. 07.093]
Zhao, H.Y., Sheng, Q.X., Lv, S.Y., et al., 2004. Characterization of three rice CCOAOMT genes. Chin. Sci. Bull., 49(15):1602-1606. [doi:10.1007/BF03184129]

\section{中文概要}

题 目: 茶树中咖啡酰辅酶 $\mathbf{A}$ 氧甲基转移酶的基因克隆及 其催化活性研究

目 的: 从茶树中克隆一种可以催化表没食子儿茶素没食 子酸酯（EGCG）生成甲基化 EGCG 的酶一一咖 啡酰辅酶 $\mathrm{A}$ 氧甲基转移酶（CCoAOMT），实现 甲基化 EGCG 的酶学合成, 为甲基化 EGCG 的进 一步开发利用提供理论依据和技术指导。

创新点: 本研究首次从茶树中克隆了一条 $C C O A O M T$ 基因 组序列; 分析了 $C C O A O M T$ 基因在不同茶树品种 和不同成熟度茶鲜叶中的基因表达规律; 证明了 CCoAOMT 具有催化合成甲基化 EGCG 的生物活 性。

方 法: 采用聚合酶链式反应 (PCR) 和序列分析获得 CCOAOMT 的编码序列和基因组序列; 采用高效 液相色谱 - 四级杆 - 飞行时间串联质谱技术 (HPLC-QTOF-MS) 分析酶促反应生成的甲基化 EGCG 产物（图 4); 采用实时苂光定量 PCR 分 析 $C C O A O M T$ 基因的表达差异（图 5）。

结 论: 本研究从茶树中克隆了 CCOAOMT 基因的编码序 列（738 bp）和基因组序列（2678 bp），明确了 该基因具有 4 个内含子和 5 个外显子; 揭示了 CCoAOMT 可以催化 EGCG 生成 EGCG4"Me、 EGCG3"Me 和 EGCG3'Me 等多种甲基化产物; 证 明了 CCoAOMT 具有催化生成甲基化 EGCG 的活 性; 并发现该基因的表达量高低与茶鲜叶的成熟 度呈正相关关系。

关键词: 茶树; 咖啡酰辅酶 $\mathrm{A}$ 氧甲基转移酶 (CCOAOMT); 催化活性; 甲基化表没食子儿茶素没食子酸酯 (EGCG) 